\title{
sur la gestion et les finances publiques L'année 2021
}

\section{Synthèse des Repères de l'année}

\section{BUDGET DE L'ÉTAT ET DES OPÉRATEURS}

\section{$\pi$ Vision stratégique et procédure budgétaire}

Une stratégie de finances publiques pour la sortie de crise

En février 2021, le Premier ministre a confié à la Cour des comptes une mission sur la stratégie de finances publiques d'après-crise. Le 15 juin 2021, la Cour répond à cette saisine en publiant un rapport dans lequel elle conclut notamment que la crise sanitaire laissera des marques durables sur l'économie française et les finances publiques. Dans ce contexte, la Cour considère que la soutenabilité de la dette publique deviendra un enjeu de souveraineté. Pour les magistrats de la rue Cambon, il s'agit d'une condition nécessaire pour faire face aux chocs économiques futurs et rester en mesure de financer les politiques et le fonctionnement des administrations publiques.

\section{La LOLF fête ses 20 ans !}

Dans une tribune publiée le 1er août 2021 dans le journal Le Monde, Alain LAMBERT et Didier MIGAUD dressent un bilan de ce texte 20 ans après sa promulgation. Bien que la LOLF ait marqué une révolution copernicienne dans la gestion de nos finances publiques, il y a, pour les « pères " de la LOLF, de nombreux motifs de déception dans la mise en application de ce texte. Selon eux, la gestion par la performance telle que résultant de la pratique de la LOLF ne permet pas une véritable gestion en fonction des résultats. La responsabilisation des gestionnaires publics apparaît également encore insuffisante, malgré les ambitions très fortes de la LOLF sur ce point. Les 20 ans de la LOLF sont également l'occasion pour eux de rappeler l'importance de la mise en œuvre d'une stratégie pluriannuelle des finances publiques.

Loi organique du 28 décembre 2021 relative à la modernisation de la gestion des finances publiques

Cette loi organique a été promulguée le 28 décembre 2021 et publiée au Journal officiel du 29 décembre 2021. Dans sa décision du 23 décembre, le Conseil constitutionnel avait validé les grandes lignes de ce texte. Les dispositions de cette loi s'articulent autour de trois axes: le renforcement du pilotage pluriannuel des finances publiques; I'amélioration de la transparence des finances publiques ; la rationalisation et l'amélioration du travail parlementaire (voir détail dans les Repères de Décembre 2021). La plupart de ses dispositions seront applicables aux lois de finances portant sur l'année 2023 (cf. Repères de Décembre 2021). La loi organique étend notamment les avis du Haut Conseil des finances publiques (HCFP) à la " cohérence » de l'article liminaire des projets de lois de finances et de financement de la sécurité sociale au regard de l'objectif pluriannuel de dépenses fixé dans la LPFP et des prévisions de recettes, de dépenses et de solde des projets de lois au regard des prévisions économiques connues. Plusieurs dispositions non organiques sur le HCFP font l'objet d'une loi ordinaire du 6 décembre 2021 portant diverses dispositions relatives au Haut Conseil des finances publiques et à l'information du Parlement sur les finances publiques.

Le Haut Conseil a adopté un avis relatif aux projets de lois de finances et de financement de la sécurité sociale pour l'année 2022

Le 22 septembre 2021, le Haut Conseil des finances publiques a publié son avis du $17 \mathrm{sep}$ tembre 2021 relatif aux projets de lois de finances 
et de financement de la sécurité sociale pour l'année 2022. Pour commencer, le Haut Conseil relève que le projet de loi de finances pour 2022 dont il a été saisi est incomplet. En effet, il n'intègre pas l'impact de mesures d'ampleur qui ont pourtant déjà été annoncées par le Gouvernement (grand plan d'investissement, revenu d'engagement notamment) et que celui-ci prévoit de faire adopter par voie d'amendement au cours du débat parlementaire. Le Haut Conseil rappelle aussi que la LPFP 2018-2022 constitue une référence dépassée pour apprécier la trajectoire de finances publiques. II note que le poids des dépenses publiques dans le PIB serait en 2022 de près de deux points au-dessus de son niveau de 2019 en dépit du net rebond de l'activité et de l'extinction de la plupart des dépenses engagées pour répondre à la crise sanitaire. De surcroît, les mesures d'allègement d'impôts des dernières années devraient peser durablement sur les recettes. Face à un endettement public accru (114 points de PIB en 2022, soit 17 points de plus qu'en 2019), le Haut Conseil appelle à la plus grande vigilance budgétaire.

\section{$\lambda$ Relations entre l'État et les opérateurs}

Dans un rapport publié le 27 janvier 2021, la Cour des comptes a présenté les conclusions d'une enquête menée sur les relations entre l'État et ses opérateurs, près de quinze ans après l'installation de cette notion en loi de finances, et quatre ans après ses derniers travaux d'ensemble sur le sujet. La Cour a relevé un effort significatif pour limiter le nombre des opérateurs. Ces derniers ont vu leur nombre diminuer de $25 \%$ sur la période 2010-2020, en grande partie sous l'effet de regroupements.

\section{Comptabilité PUBliQue}

\section{$\pi$ Juridictions financières}

Projet stratégique des juridictions financières « JF2025 "

Le jeudi 4 février 2021, le Premier président de la Cour des comptes Pierre Moscovici a présenté à la presse un nouveau projet stratégique des juridictions financières baptisé «JF2025 ». Le rapport « JF 2025 » s'organise autour de 12 orientations destinées à rendre les travaux des juridictions financières plus diversifiés et accessibles, à moderniser les métiers du juge financier et à rendre plus agile et intégré le fonctionnement des juridictions financières. Par cet ambitieux projet « JF2025 », Pierre Moscovici souhaite ainsi parachever l'œuvre engagée sous la présidence de Philippe Séguin.

Vers une responsabilité unifiée des gestionnaires publics

Le projet de loi de finances 2022, en son article 41 , habilite le Gouvernement à prendre par voie d'ordonnance toute mesure permettant d'harmoniser le régime juridictionnel de responsabilité des gestionnaires publics, passant notamment par une révision du code des juridictions financières. L'objectif de la réforme est de définir un nouveau régime unifié de responsabilité applicable à l'ensemble des agents publics, comptables comme ordonnateurs, plus simple, plus lisible et plus juste. II vise à sanctionner plus efficacement les fautes graves relatives à l'exécution des recettes et des dépenses ou à la gestion des biens provoquant des préjudices financiers significatifs. II s'agit de réserver l'office du juge aux cas d'une gravité avérée et non pas de sanctionner des fautes purement formelles. Dans ce cadre, la responsabilité personnelle et pécuniaire du comptable public sera abrogée et la Cour de discipline budgétaire et financière supprimée (cf. Repères de Décembre 2021).

\section{FinANCES LOCALES}

\section{Les finances des collectivités locales en 2021}

Cette note de la DGCL était particulièrement attendue pour analyser la nature de la reprise tant annoncée du côté des collectivités territoriales après une année terrible de baisse de l'investissement. En termes de dépenses de fonctionnement, celles-ci augmentent de $+2,4 \%$ avec une hausse concernant surtout les achats et les charges externes. L'épargne brute continue à diminuer avec une baisse de - 3,9\% au budget primitif 2021 après une baisse de - 10,8 \% en 2020 . Les recettes d'investissement augmenteraient, pour leur part, plus fortement $(+10,4 \%$ ) que les dépenses d'investissement.

L'analyse financière des communes et des $\mathrm{EPCl}$ en 2020 par l'Association des maires de France et la Banque des territoires

Avec cette nouvelle analyse, I'impact financier de la crise commence à être mesuré correctement avec un effet ciseau différencié selon les strates de collectivités. De façon générale, les dépenses ont stagné en 2020 avec cependant des variations internes. Ainsi, les dépenses d'intervention augmentent de $+2,1 \%$ tandis que les achats et 
charges externes ont diminué de - 4,8\% entre 2020 et 2019 en raison de la fermeture d'un certain nombre de services publics (cf. Repères de Novembre 2021 pour plus de précision).

\section{Les intercommunalités et la relance}

Le Premier ministre et le président de l'Assemblée des communautés de France (ADCF), ont signé le 22 mars 2021 un accord de partenariat concernant la mise en œuvre des contrats de relance et de transition écologique (CRTE). Selon le communiqué de presse publié, « les CRTE ont été conçus pour contribuer à la mise en œuvre de plusieurs mesures du plan France Relance au plan local et pour accompagner les grandes transitions économiques, écologiques et de cohésion à l'œuvre dans tous les territoires. Ils ont vocation à demeurer évolutifs et s'inscrire dans la durée du mandat municipal 2020/2026».

\section{Regard financier sur les départements}

Dans cette étude, l'Association des départements de France (ADF) constate que les dépenses d'action sociale ont augmenté dans la part des dépenses de fonctionnement des départements. Elles représentaient $47 \%$ en 2001, $58 \%$ en 2004 et $62 \%$ en 2019. Parmi ces dépenses, le RSA occupe une place à part en raison de la hausse significative du nombre de bénéficiaires en 2020, pour partie compensée par des baisses au premier semestre 2021. Par ailleurs, les recettes de fonctionnement ont augmenté grâce à des impôts indirects devenus prépondérants en 2021. On peut aussi noter que les DMTO ont globalement bien résisté en 2020 avec des évolutions hétérogènes selon les collectivités. Cette étude note enfin une reprise de l'investissement depuis 2018, du fait de subventions nationales et européennes.

\section{Rapport de la Cour des comptes sur les aides à l'investissement local}

La Cour des comptes a publié en mai 2021 un référé au Premier ministre sur Les concours financiers de l'État en soutien à l'investissement public local. Dans un contexte de relance de l'investissement, cette analyse des juges financiers prône la remise en cause de certains aspects. En premier lieu, la Cour des comptes alerte sur l'importance de doter le comptable des moyens de contrôler le FCTVA. En second lieu, les sages attirent l'attention sur le fait que les subventions profitent principalement aux grandes aires urbaines délaissant un certain nombre de territoires. Enfin, la Cour des comptes relève que la démarche de subventions devrait davantage s'inscrire dans une logique de pluriannualité et de performance.

\section{FinANCES SOCIALES}

\section{$\pi$ Situation des comptes sociaux et conséquences de la crise sanitaire}

Les comptes de la protection sociale pour 2020 et la progression très sensible des dépenses

La DREES a publié le 15 décembre le rapport sur la protection sociale en France et en Europe en 2020. (NB : les comptes de la protection sociale couvrent un périmètre sensiblement plus large que celui des lois de financement de la SS). En France, du fait de la crise, les dépenses de protection sociale ont augmenté de 7,8 \% en 2020, soit un accroissement de $46 \mathrm{Md}$. Les ressources de la protection sociale $n^{\prime}$ ont diminué que de $0,1 \%$, les compensations massives de l'État aux pertes de ressources des différents régimes, principalement pour l'activité partielle, ayant neutralisé les pertes. De fait, le déficit du compte de la protection sociale a atteint -51Md€. C'est en France que la part des dépenses de protection sociale par rapport au PIB est la plus forte (35\% du PIB et $12080 €$ par habitant), notre pays se situant au $4^{\mathrm{e}}$ rang en Europe (sur 20 États) pour la dépense par habitant, derrière le Danemark, l'Autriche et l'Allemagne. Par ailleurs, les prestations de lutte contre la pauvreté et l'exclusion ont augmenté fortement ( $+12 \%$ en France, du fait en particulier du RSA). Les dépenses au titre du risque «vieillesse » ont ralenti leur progression, celles des prestations familiales ont diminué, du fait notamment du moindre recours aux gardes d'enfant.

Le rapport de la Cour des Comptes sur le bilan opérationnel de l'utilisation des dépenses publiques pendant la crise

La Cour des Comptes a rendu le 9 septembre 2021 le rapport demandé par la Commission des finances de l'Assemblée Nationale sur le bilan opérationnel de l'utilisation des dépenses publiques pendant la crise. La Cour considère notamment que les objectifs poursuivis - notamment pour le soutien aux entreprises - ont été globalement atteints à court terme, grâce à des dispositifs souvent plus favorables que chez nos partenaires, mais avec les risques d'effets d'aubaine et de fraude significatifs, en particulier pour le Fonds de solidarité et l'activité partielle. La Cour soulève la question de l'efficience de ces aides sur le long terme dans la mesure où elles ont conduit à aggraver sensiblement l'endettement des entreprises. S'agissant de l'objectif du soutien à l'emploi, les dispositifs ont permis de contenir le chômage, sauf pour les salariés en 
contrats précaires. Enfin le revenu des ménages a globalement été bien préservé puisqu'il a même augmenté de $1 \%$ en 2020, alors que le PIB reculait de $8 \%$.

\section{Dette sociale, loi de financement de la Sécurité sociale et révision de la LOLFSS}

\section{Le rapport de la Cour des Comptes sur la Sécurité Sociale}

La Cour des Comptes a publié le 6 octobre son rapport annuel sur l'application des lois de financement de la Sécurité sociale jusqu'en 2020. Elle part du constat que la crise du Covid19 bouleverse la trajectoire financière de la Sécurité sociale, puisqu'en 2020 le déficit agrégé des régimes obligatoires de base et du FSV atteint $1,7 \%$ du PIB, que l'amélioration prévue pour 2021 sera très limitée du fait du maintien à un niveau très élevé des dépenses de santé (cf. tests, vaccination, montée en puissance du Ségur de la Santé pour $+7,9 \mathrm{Md}$ ), et que pour les années à venir le déficit ne pourra pas être inférieur à $13 \mathrm{Md} €$ en raison du poids des dépenses de l'Assurance maladie (+12,5 Md€ au titre du Ségur de la santé en 2022). De ce fait, selon la Cour, le plafond de 92Md€ d'autorisation de reprise de dette par la CADES pour la période 2020-2023 ne devrait pas permettre de couvrir le déficit de 2023. Au 31 décembre 2021, la CADES aura amorti 205 Md€ de dette sociale sur les 320 Md€ repris depuis sa création, et les programmes de 2021-2022 s'inscrivent dans la trajectoire de reprise des 136 Md€ prévus par la loi d'août 2020 (cf. Repères d'Octobre 2021 pour plus de détail).

Proposition de loi organique au Sénat pour le renforcement du contrôle du Parlement sur les finances sociales

Le rapporteur général de la Commission des affaires sociales du Sénat a déposé, le 6 avril, une proposition de loi organique visant à renforcer le contrôle du Parlement sur les finances sociales. Elle comporte 4 axes : faire coïncider le périmètre des LFSS avec celui des lois de programmation des finances publiques (extension à I'UNEDIC et aux régimes de retraite complémentaires ; renforcer la clarté et la normativité des LFSS (extension du caractère limitatif de certaines dépenses) ; étendre le contrôle du Parlement en créant une loi d'approbation de comptes de la sécurité sociale; instaurer une « règle d'or » garantissant sur cinq ans l'équilibre des comptes de la Sécurité sociale.

\section{Mesures de soutien contre la crise}

La prolongation des aides pour les secteurs qui continuent d'être impactés par la crise

À la suite de l'annonce par le Premier Ministre, le 9 décembre 2021, de la poursuite des restrictions d'activité directes ou indirectes pour certains secteurs (ex. traiteurs, agences de voyage, événementiel), les ministres de l'économie et du travail ont confirmé la prolongation des dispositifs d'aides (cf. Repères de Décembre 2021 pour plus de détail).

\section{त Dépenses de santé/Hôpital/ Assurance maladie}

Le bilan du "Ségur de la Santé ", un an après son lancement

Le ministère des solidarités et de lasSanté a publié le 20 juillet un dossier de presse pour faire le bilan du "Ségur de la Santé » lancé le 13 juillet 2020 par le Premier ministre. Ce plan a comporté un programme de $9 \mathrm{Md}$ f pour la revalorisation des métiers. Ces revalorisations, qui englobent en particulier le «socle » de $183 €$ nets mensuels accordés à tous les personnels et des révisions des grilles indiciaires propres à chaque catégorie de soignants et de personnel médical et non médical, sont détaillés dans le dossier. Par ailleurs, dans la continuité de la stratégie "Ma santé 2022 » de 2018, le plan accélère la réduction de la part de la tarification à l'activité (T2A) dans le financement des établissements (cf. Repères de Juillet 2021).

Un nouveau bras de fer sur la contribution financière des complémentaires santé

Selon des informations parues dans Les Échos du 21 octobre, le bras de fer est reparti entre le gouvernement et les organismes complémentaires de santé (OCS). Ceux-ci s'étaient vu imposer en 2020 une contribution de 1,5 Md€ au motif qu'ils avaient fait des économies du fait du moindre recours aux soins pendant la pandémie et pour contribuer au redressement financier de la Sécurité sociale. Les ministres Olivier Véran et Olivier Dussopt leur ont écrit pour leur signifier que le niveau des économies constatées avait été encore supérieur de 500 M€ en 2020 et qu'il conviendrait de le restituer à leurs adhérents sous forme d'un gel des tarifs en 2022. Cela a provoqué de vives réactions de la part des OCS, qui ont fait valoir qu'ils avaient subi le choc des reports de soins en 2021 ainsi que de la réforme de la prise en charge à $100 \%$ de certaines dépenses. Ce bras de fer intervient dans un climat animé par les réflexions 
sur la "Grande Sécu » contre laquelle les OCS sont vent debout.

\section{$\lambda$ Retraites et Dépendance}

Le Président de la République réoriente les perspectives de réforme des retraites

Dans son entretien à TF1 le 15 décembre, le Président de la République a rappelé la nécessité de reprendre le chantier de la réforme des retraites dès 2022, en insistant sur le fait qu'il faudrait travailler plus longtemps «sans que cela signifie la même réalité pour tous 》. Au-delà du «Régime universel », il a envisagé de remplacer les 42 régimes actuels par «trois grands régimes » pour les salariés du privé, pour le secteur public et pour les indépendants.

L'accord sur les mesures de redressement de I'AGIRC-ARRCO

Selon les informations parues dans Les Échos, pour faire face au déficit de l'AGIRC-ARRCO (voir Repères de Juin), le patronat et deux syndicats (CFDT et CFFTC) se sont mis d'accord pour autoriser le Conseil d'administration à sous-indexer (jusqu'à 0,5 points) la revalorisation des pensions par rapport à l'inflation en 2021 et 2022.

Le rapport de la Cour des comptes sur la prévention de la perte d'autonomie des aînés

Le rapport thématique public de la Cour des Comptes sur la prévention de le la perte d'autonomie part du constat que si l'espérance de vie à 65 ans est, en France, supérieure à la moyenne européenne, l'espérance de vie en bonne santé ne représente que la moitié seulement de cette durée espérée; selon la Cour, un an d'espérance de vie sans incapacité ferait économiser 1,5 Md€ à l'assurance maladie. L'organisation de la prise en charge de la dépendance est très complexe, avec de multiples intervenants (ex. Caisses de retraite, départements, ARS, Assurance maladie, mutuelles, bailleurs sociaux) même si la loi du 28 décembre 2015 sur « l'adaptation de la société au vieillissement » a institué une " conférence des financeurs publics ». II s'ensuit des divergences d'approche sur la mise en œuvre de certains aspects essentiels de la politique de prévention, comme le dépistage des pathologies du grand âge (cf. Repères de Novembre 2021 pour plus de détail).

\section{त Solidarité/Lutte contre la pauvreté/ Revenu universel/Pouvoir d'achat}

La forte augmentation du nombre d'allocataires au RSA en 2020
Selon les statistiques provisoires de la CNAF, le nombre d'allocataires du RSA devait être de 2,06 millions en décembre 2020, soit une progression de 7,5\% sur un an. Cette forte augmentation fait suite à une longue période de stabilité qui avait duré de janvier 2017 à février 2020, et est due plus à des « rechutes » d'anciens allocataires qu'à l'entrée de nouveaux bénéficiaires, ainsi qu'à la réduction du nombre de sorties. La Prime d'activité a peu progressé, avec 4,58 millions de foyers bénéficiaires en décembre 2020, soit une augmentation de $2,1 \%$ en un an.

Le Conseil d'État appelle à plus de cohérence pour la prise en compte des ressources ouvrant droit aux prestations sociales sous conditions

Dans une étude rendue au Premier ministre le 10 novembre, le Conseil d'État fait le constat d'une très grande hétérogénéité pour l'appréciation de la «base ressources » qui détermine l'éligibilité et le calcul des prestations sociales attribuées sous conditions. Une trentaine de prestations sont ainsi concernées, pour un montant de $120 \mathrm{Md}$ : les aides au logement (17 Md€), le RSA (11,3 Md€), la PAJE (11 Md€), I'AAH $(9,7 \mathrm{Md})$, auxquelles s'ajoutent l'aide sociale légale des départements, des prestations facultatives servies par les collectivités et les mécanismes de tarification pour l'accès à des services publics (ex. cantines, crèches). L'objectif est de clarifier et harmoniser une situation dont l'illisibilité pénalise lourdement les ayants droit et favorise le non recours aux prestations (cf. Repères de Novembre 2021 pour plus de détails).

Une étude de I'Institut des politiques publiques sur les gains de pouvoir d'achat

La question de l'évolution du pouvoir d'achat et de sa distribution pendant le quinquennat, et plus particulièrement depuis les 18 mois de la crise sanitaire, fait l'objet de nouvelles controverses. Plusieurs analyses -dont celle de l'INSEE, celle de de la Direction générale du Trésor associée au rapport économique, social et financier du PLF pour 2022 - ont conclu qu'entre 2017 et 2022, le revenu disponible des ménages aura globalement progressé, avec une forte concentration sur tranches les moins aisées de la population (+ $10 \%$ ) contre $+2 \%$ pour les plus aisées. Mais, selon La note de I'IPP, I'Institut des Politiques publiques, s'il y a eu un gain moyen de $1,6 \%$ du revenu disponible des ménages, ce gain serait inférieur à $1,5 \%$ pour les tranches les moins aisées et de l'ordre de 2,8\% pour les $1 \%$ personnes les plus aisés (et jusqu'à $+4,1 \%$ pour les $0,1 \%$ les plus fortunés). Les catégories les 
moins aisées ont bénéficié de mesures à caractère social durant la crise (ex. revalorisation de la prime d'activité, chèque énergie), mais d'autres décisions ont joué en sens contraire (ex. baisse $\operatorname{des} A P L)$, si bien que la situation est hétérogène au sein de ces catégories. En revanche, il semble que les mesures fiscales (ex. création du prélèvement forfaitaire unique, suppression de I'ISF, suppression de la taxe d'habitation) ont pu avoir des effets croissants pour les catégories les plus aisées.

\section{$\lambda$ Famille, démographie et jeunesse}

Les mesures pour faire face à la précarité des jeunes se multiplient

Particulièrement préoccupé par la dégradation de la situation des jeunes, et tout spécialement des étudiants, le gouvernement, au-delà des mesures du plan « 1 jeune, 1 solution » prolonge les aides de soutien au revenu mises en place à I'automne et en annonce d'autres: augmentation des bourses, repas à $1 €$, gel des loyers des résidences universitaires, doublement des fonds d'urgence, institution d'une prime de $1000 €$ pour le premier logement pour les jeunes actifs. II a repris quelques-unes des mesures préconisées dans le rapport du Conseil d'orientation des politiques de jeunesse de février 2021 sur l'évolution de la Garantie jeunes en élargissant des critères d'éligibilité, notamment sur les plafonds de ressources et en augmentant la durée d'accompagnement. Le gouvernement espère ainsi résister à une pression croissante en faveur de l'élargissement du bénéfice du RSA aux moins de 25 ans.

Le premier bilan de la réforme des aides publiques au logement

Le ministère de la transition écologique a publié le 22 juillet un premier bilan de la très controversée réforme des $A P L$ qui est entrée en vigueur au 1 er janvier 2021 et qui a visé à calculer les APL sur la base des revenus des 12 derniers mois, au lieu de prendre en compte ceux de l'année N-2, sans autre modification des paramètres de calcul. Selon le ministère, la réforme a conduit, sur les près de 6 millions d'allocataires, à diminuer l'allocation de janvier 2021 pour 29,6\% d'entre eux, à l'augmenter pour $18,2 \%$ et à la maintenir à I'identique pour 52,2\%. L'économie réalisée sera finalement supérieure à ce qu'escomptait le gouvernement, soit un montant de dépenses inférieur de 1,1 Md€ à celui de 2020 (contre 0,75 Md€ prévus en LFI 2021.)

Le rapport du Haut-Commissariat au plan sur la démographie française

Le Haut-Commissariat au plan a publié en mai 2021 une note sur le devenir de la démographie française. Au-delà des aspects les plus commentés sur le recours à l'immigration, ce rapport a rappelé que cette problématique était essentielle pour l'avenir de notre système de protection sociale, puisque le maintien du contrat social français fondé sur la redistribution et la solidarité entre générations dépend de la structure équilibrée de la population. Le modèle français de politique familiale, avec ses diverses composantes (ex. quotient familial, prise en charge précoce de la petite enfance) a permis le maintien d'un taux de fécondité exceptionnel en Europe. Mais un ralentissement de l'évolution du solde naturel s'est amorcé depuis 2010. Pour autant, le recours à l'immigration n'est pas la solution univoque pour pallier financièrement le ralentissement de la démographie en raison du coût des politiques sociales d'accompagnement induites. Le Haut-Commissariat conclut à la nécessité de revoir tous les leviers de la politique familiale dans le cadre d'un « Pacte national pour la démographie ».

\section{$\lambda$ Assurance chômage}

L'amélioration de la situation de l'emploi permet au gouvernement d'escompter l'entrée en vigueur de la réforme de l'assurance chômage au $1^{\text {er }}$ octobre 2021

Les deux conditions de « retour à meilleur fortune » auxquelles est subordonnée l'entrée en vigueur de la réforme de l'Assurance chômage - atteinte d'un seuil minimal de déclarations d'embauche et baisse du nombre des demandeurs d'emploi inscrits à Pôle Emploi - semblent en passe d'être satisfaites (Cf. Repères de Juin et d'Octobre 2021) : en effet le nombre cumulé sur 4 mois des déclarations d'embauche a dépassé de 100000 l'objectif visé de 2,7 millions et, d'autre part, le nombre des chômeurs inscrits à Pôle emploi a baissé de 172000 en 6 mois. Dans ces conditions, le gouvernement pourrait réussir à faire passer sa réforme devant le Conseil d'État. Elle serait appliquer à partir du 1er octobre avec, en particulier, de nouvelles modalités de calcul de l'indemnisation, moins avantageuses que par le passé.

\section{Quels effets de la réforme de l'assu- rance-chômage pour I'UNEDIC ?}

Selon les informations contenues (p. 131) dans le rapport économique, social et financier annexé au PLF 2022, le gouvernement attend de la réforme de l'assurance-chômage 1,9Md€ d'économies en 2022, qui contribueraient à ramener le Régime à l'équilibre dès 2022 (contre un déficit de - 11,9 Md€ en 2021). L'endettement de I'UNE- 
DIC se situerait à $66 \mathrm{Mds€}$ en 2022. Ces prévisions paraissent confortées par les statistiques de l'emploi qui escomptent un taux de chômage au sens du BIT stabilisé à 7,6\% à la fin de 2021 selon I'INSEE. Les nouvelles prévisions financières avalisées par le bureau de I'UNEDIC du 22 octobre confirment un déficit de -10Md€ en 2021, puis un excédent de 1,5 Md€, et de 2,3 Md€ en 2023 ; cette amélioration serait la résultante de trois facteurs : pour $60 \%$ la fin des mesures d'urgence, pour $25 \%$ le rebond de l'emploi et pour $15 \%$ les effets de la réforme de l'indemnisation.

\section{Fiscalité ET PROCÉDURES FISCALES}

\section{Contrôle fiscal}

La publication du rapport d'activité de la DGFIP pour 2020

La DGFiP a présenté son rapport d'activité après une année 2020 bien particulière, marquée par la crise sanitaire. Ce rapport confirme une baisse des recettes liées aux contrôles fiscaux en raison des circonstances exceptionnelles. Au total, l'État a recouvré 7,8 Mds€ en 2020, contre 11 Mds€ en 2019, mais néanmoins équivalent au montant de 2018. Le montant total des redressements établis à la suite des contrôles fiscaux a atteint 10,2 Mds€, au lieu de 13,5 Mds€ en 2019. Ils ont principalement concerné I'IS (2 Mds€) et les remboursements de crédits de TVA (1,7 Mds€). En raison de la crise liée à la covid-19, les services fiscaux se sont concentrés sur les contrôles sur pièces au détriment de ceux effectués sur place, et ils ont ciblé leurs missions de contrôle sur les dossiers patrimoniaux à forts enjeux. Ainsi, ce sont seulement 104586 contrôles sur pièces des professionnels qui ont eu lieu en 2020, contre 137095 en 2019. Et ce sont seulement 17483 contrôles qui se sont déroulés dans les locaux des entreprises, contre 35545 en 2019. Au sens large, toutes procédures fiscales confondues, ce sont un peu plus de 272000 contrôles fiscaux qui ont été menés auprès des professionnels (374 000 en 2019). Afin de faire face à la fraude fiscale, la DGFiP s'appuie enfin de plus en plus sur l'intelligence artificielle et le data mining. Près d'un contrôle sur trois a été ciblé par I'intelligence artificielle (contre seulement $22 \%$ en 2019).

\section{$\lambda$ Impôt sur le capital}

Les recettes fiscales croissantes de la taxe sur les transactions financières (TTF)
Instaurée par la loi de finances pour 2012, la TTF (CGI, art. 235 ter ZD), qui s'applique aux titres des sociétés dont la capitalisation boursière est supérieure à 1 Md€ (BOFIP, 23 déc. 2020), soit environ 134 sociétés, a généré en 2020 environ 1,785 Mds€, soit 340 M€ supplémentaires par rapport à l'exercice 2019, du fait de l'explosion des volumes de transactions boursières en 2020 .

\section{Le rendement budgétaire de l'impôt sur la fortune immobilière (IFI) en 2020}

L'IFI a rapporté 1,56 Mds€ en 2020, soit 500 M€ de moins qu'en 2019 en incluant les sommes dues au titre de I'IFI des années précédentes et de l'ancien ISF ; ou en progression de 4,4\% au regard du seul IFI qui a eu un rendement budgétaire de 1,49 Mds€ en 2019. Selon la note publiée par la DGFIP en juillet, il est possible d'observer la grande hétérogénéité des contribuables assujettis à I'IFI : un quart seulement a un patrimoine immobilier supérieur à 2,5 M€. Selon cette note, plus de 143000 foyers ont adressé en 2020 une déclaration d'IFI, en progression de $3 \%$ sur un an.

Le $3^{e}$ rapport du Comité d'évaluation des réformes de la fiscalité du capital

Le Comité d'évaluation des réformes de la fiscalité (France Stratégie, Premier ministre), a remis le 15 octobre 2021, son $3^{e}$ rapport qui vient actualiser les données chiffrées fournies dans les précédents rapports sur l'instauration du PFU et la transformation de I'ISF en IFI, ce qui permet de dresser quelques enseignements. Le rapport souligne que l'instauration de la "flat tax" a pu conduire à une hausse globale de l'investissement des entreprises. Selon le Comité, les réformes menées dans le cadre de la loi de finances pour 2018 ont conduit à ramener les taux d'imposition de la France sur les actifs mobiliers à des niveaux proches de la moyenne observée dans les principaux États comparables. Auparavant, le taux d'imposition marginal effectif des placements financiers pouvait dépasser $100 \%$ pour les contribuables assujettis à I'ISF, ce qui était « dissuasif » note le rapport. La forte progression des dividendes déclarés par les ménages au titre de 2018 (23 Mds€ contre 14 Mds€ en 2017) s'est confirmée en 2019 (24 Mds€) et en 2020 (24 Mds€), ce qui a permis d'alimenter les caisses de l'État en recettes fiscales, alors qu'à l'inverse, la soumission des revenus du capital au barème de l'impôt sur le revenu en 2013 s'était traduite par une perte nette. Le passage de I'ISF à I'IFI, a permis une baisse du nombre d'expatriations fiscales et une hausse du nombre d'impatriations fiscales de ménages français fortunés, 
même si le revenu médian des « revenants » déclarés reste toutefois inférieur à celui des « partants » : $94000 €$ contre $156000 €$ pour 2019. Si le passage de I'ISF à l'IFI a représenté une perte de recettes fiscales d'environ $3 \mathrm{Mds} €$, le coût de la réforme est bien inférieur aux prévisions, environ 1,5 Mds€, sans compter les autres recettes fiscales liées aux retours non évaluées à ce jour (ex. IR, TH, TFPB).

\section{Le niveau élevé des taxes foncières sur les propriétés bâties?}

Selon I'Union nationale des propriétaires immobiliers (UNPI), la taxe foncière sur les propriétés bâties, hors taxe d'enlèvement des ordures ménagères (TEOM) et taxe foncière sur les propriétés non bâties (TFPNB), aurait connu une augmentation de près de $28 \%$ entre 2010 et 2020, dont $11,5 \%$ de hausse au cours des cinq dernières années (35,3 Mds€ collectés en 2020). En mai 2021, une étude de l'Association des maires de France a confirmé cette tendance haussière. La revalorisation nationale des valeurs locatives cadastrales, pour les locaux professionnels, et les hausses de taux décidées localement expliquent principalement cette évolution. Pour les plus petites communes, il s'agit de compenser les pertes liées à la suppression programmée de la taxe d'habitation sur les résidences principales.

\section{$\pi$ Impôt sur les sociétés}

\section{La France continuera à prélever la taxe GAFAM jusqu'en 2023}

L'administration fiscale a annoncé continuer à prélever la taxe GAFAM jusqu'en 2023. Celle-ci ne disparaîtrait que lorsque la grande réforme de l'impôt mondial conduite par l'OCDE serait effective. Le ministre de l'économie et des finances s'est dit « prêt à prendre un engagement formel vis-à-vis des États-Unis » sur la disparition de la taxe dont le produit était 375 M€ en 2020, soit près de $100 \mathrm{M} €$ de plus qu'en 2019. Dans I'attente, le calendrier de versement de la taxe GAFAM des acomptes est resté inchangé en 2021.

Accord sur de nouvelles règles internationales concernant la fiscalité des entreprises

Après le sommet du G7 du 5 juin 2021, 130 États de I'OCDE (90\% du PIB mondial) sont parvenus le 10 juillet 2021 à un accord sur une réforme de la taxation des multinationales, prévoyant notamment la mise en place d'un impôt minimum sur les bénéfices d'au moins $15 \%$. Ces États ont adhéré à la Déclaration qui instaure un nouveau cadre pour la réforme de la fiscalité internationale. Les éléments restants du cadre de la réforme, y compris le plan de mise en œuvre, seront finalisés en octobre 2021, pour une mise en $œ u v r e$ effective en 2023. Le directeur de la fiscalité à l'OCDE Pascal Saint-Amans pense que cet accord signe «la fin des paradis fiscaux ». Même la Suisse y a adhéré, " sous certaines conditions». La Commission européenne s'est félicitée de cette avancée du droit international fiscal. Le cadre de référence établi repose sur deux piliers qui visent à faire en sorte que les grandes entreprises multinationales paient des impôts là où elles exercent des activités et génèrent des bénéfices, tout en renforçant la sécurité juridique et la stabilité du système fiscal international. Le premier pilier garantit une répartition plus équitable des bénéfices et des droits d'imposition entre États concernant les grandes entreprises multinationales, y compris celles du numérique. Il permettra de réattribuer une partie des droits d'imposition sur les entreprises multinationales de leurs États d'origine aux États de marché dans lesquels elles exercent des activités commerciales et réalisent des bénéfices, qu'elles y aient ou non une présence physique. Des droits d'imposition sur plus de 100 Mds $\$$ de bénéfices devraient être réattribués chaque année aux juridictions de marché. Le second pilier encadre la concurrence en matière d'impôt sur les bénéfices des sociétés en introduisant un impôt minimum mondial que les États peuvent prélever pour protéger leur base d'imposition. Avec un taux d'au moins $15 \%$, l'impôt minimum mondial devrait générer environ $150 \mathrm{Mds} \$$ de recettes fiscales supplémentaires par an au niveau mondial. La définition de la base taxable demeure un enjeu majeur, qui préoccupe les grandes entreprises françaises

\section{ク TVA}

\section{L'ordonnance relative à la généralisation de la facture électronique}

Après que la DGFIP ait soumis à consultation publique du 13 août au 13 octobre 2021 ses commentaires de la transposition du paquet TVA dit «commerce électronique » (v. Repères d'Août 2021), le Gouvernement vient de prendre une ordonnance relative à la généralisation de la facturation électronique, jusqu'alors facultative, dans les transactions entre assujettis à la TVA et à la transmission des données de transaction (Ord. $n^{\circ}$ 2021-1190 du 15 sept. 2021, JORF n 0216, 16 sept. 2021) sur habilitation donnée par le législateur pour ce faire (art. 195 L. n 2020-1721 du 29 déc. 2020 de finances pour 2021). Un calen- 
drier graduel est prévu pour la mise en œuvre de cette nouvelle obligation d'émission des factures sous forme électronique (CGI, art. 289-VI) : $1^{\text {er jan- }}$ vier 2024 pour les grandes entreprises ; 1 er janvier 2025 pour les entreprises de taille intermédiaire ; 1er janvier 2026 pour les PME et les TPE.

\section{MANAgement PUBLIC}

\section{$\pi$ Gestion publique}

Le Rapport public annuel 2021 de la Cour des comptes

Si la Cour a choisi de consacrer la première partie de son rapport public annuel (RPA) 2021 à différents thèmes directement liés à cette crise (cf. partie Finances sociales), la seconde partie du RPA propose, plus classiquement, un échantillon représentatif des travaux, tant de la Cour que des chambres régionales et territoriales des comptes.

\section{L'évaluation des politiques publiques}

Le Cercle de la Réforme de l'État a publié le 13 septembre 2021 une note sur l'évaluation des politiques publiques « au service de la réforme de l'État : réconcilier la connaissance et l'action, opérationnaliser l'évaluation". Ce document d'une douzaine de pages vise à ce que l'évaluation "ne soit pas perçue comme une activité d'experts [mais qu'elle] réponde effectivement aux attentes de la société ».

\section{$\pi$ Fonction publique}

La place croissante des contractuels dans la fonction publique en 2019

Selon une analyse de l'INSEE publiée au mois de mars 2021, 5,66 millions de salariés (1 salarié sur 5) travaillaient dans la fonction publique fin 2019 , soit 18300 de plus que fin 2018 (+ 0,3\%). Sur la même période, on constate que le nombre de fonctionnaires poursuit sa baisse $(-0,3 \%)$ tandis que le nombre de contractuels augmente de 5,2 \% (55 600 personnes dont 25400 d'emplois aidés). Au total, les contractuels représentaient 19,9\% des emplois à la fin de l'année 2019.

\section{Les derniers concours de I'ENA}

89 places sont offertes aux concours 2021 de l'École nationale d'administration (ENA), contre 83 l'année dernière, selon un arrêté du 20 juillet 2021. Pour rappel, il s'agit des derniers concours que l'ENA organisera puisqu'elle sera remplacée

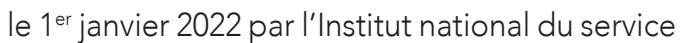
public (INSP). La hausse du nombre de places s'explique par le concours externe dit «Talents» réservé aux personnes d'origine modeste. En revanche, les nombres de places offertes pour les autres concours de l'ENA restent les mêmes qu'en 2020 : 40 pour le concours externe, 32 pour le concours interne, 7 pour le troisième concours et 4 pour le concours réservé aux docteurs.

\section{Réforme des inspections générales}

Le Gouvernement a transmis aux organisations syndicales le 27 avril 2021 l'article 7 du projet d'ordonnance de réforme de la haute fonction publique. Ce texte, relatif aux inspections générales, prévoit la mise en extinction de trois corps : inspection générale des finances (IGF), inspection générale de l'administration (IGA) et inspection générale des affaires sociales (IGAS). $\mathrm{La}$ «fonctionnalisation » de ces corps impliquera des affectations à durée limitée.

\section{$\pi$ Immobilier public}

La direction de l'immobilier de l'État a publié le 22 juin 2021 son rapport L'immobilier public de demain. Ce document souligne que la crise sanitaire a modifié le rapport que les agents ont avec leur lieu de travail, dans le contexte d'une banalisation du télétravail.

\section{$\pi$ Collectivités territoriales}

\section{Les propositions du CNEN en vue d'un nouveau " pacte territorial »}

Le Conseil national d'évaluation des normes (CNEN) vient de publier un rapport relatif à «l'intelligibilité et à la simplification des normes applicables aux collectivités territoriales au service de la transformation de l'action publique ». Parmi les réflexions conduites pour fluidifier les relations entre l'État et les collectivités territoriales, le CNEN recommande de renforcer la portée du principe d'autonomie. II s'agit « d'aiguiller le législateur dans l'élaboration des projets de texte applicables aux collectivités territoriales » en précisant, au niveau constitutionnel (et organique), le contenu précis de la notion de libre administration.

\section{Simplification des expérimentations lo- cales}

Le Conseil constitutionnel a validé le 15 avril 2021 (décision 2021-816 DC) la loi organique relative à la simplification des expérimentations mises en œuvre sur le fondement du quatrième alinéa de I'article 72 de la Constitution. Celle-ci avait été adoptée le 16 mars 2021 par le Parlement suite à un vote conforme par les députés du texte adopté en première lecture par le Sénat le 3 novembre 2020. La loi (dossier complet) prévoit notamment: de simplifier la procédure d'entrée 
dans l'expérimentation; d'assurer une évaluation plus pertinente des expérimentations; de permettre la sortie de l'alternative binaire entre la généralisation ou l'abandon de l'expérimentation nonobstant le rappel par le Conseil du principe d'égalité.

\section{EUROPE}

\section{$\pi$ Euro}

BCE : maintien d'une politique monétaire accommodante

Le 16 décembre 2021, le conseil des gouverneurs de la Banque centrale a estimé que « les progrès en termes de reprise économique et vers son objectif $d$ 'inflation à moyen terme autorisent une réduction graduelle du rythme de ses achats d'actifs au cours des trimestres à venir. Mais le soutien monétaire reste nécessaire en vue de la stabilisation de l'inflation au niveau de son objectif de $2 \%$ à moyen terme ». Le taux d'intérêt des opérations principales de refinancement ainsi que ceux de la facilité de prêt marginal et de la facilité de dépôt demeurent inchangés, à respectivement 0,00\%, $0,25 \%$ et - 0,50 \%. Une politique considérée par la presse comme accommodante voire très accommodante.

\section{Eurogroupe : la pandémie a exacerbé les} déséquilibres économiques

Lors de leur réunion en visioconférence du 18 janvier 2021, en se fondant sur une note technique élaborée par la Commission européenne, l'Eurogroupe a tenu un débat thématique sur les déséquilibres macroéconomiques dans la zone euro, exacerbés par la crise de la COVID-19. Ils ont discuté des stratégies politiques nécessaires pour y faire face.

\section{Les vingt ans de l'Euro}

Le 31 décembre 2021, les ministres des finances de l'Eurogroupe ont publié une tribune conjointe pour célébrer les 20 ans de la diffusion de la monnaie numéraire en euro. Dressant un bilan favorable, ils ont notamment annoncé une révision des règles budgétaires communes.

\section{Signature de l'accord modifiant le traité instituant le MES}

Le 27 janvier 2021, les représentants des États membres ont signé les accords du 30 novembre 2020 modifiant le traité instituant le mécanisme européen de stabilité et l'accord intergouvernemental concernant le fonds de résolution unique, ce qui lance les procédures de ratification nationales. Cet accord permet notamment au MES de fournir un filet de sécurité commun pour le fonds de résolution unique au moyen d'une ligne de crédit à partir du début de 2022, soit deux ans avant la date prévue.

\section{$\pi$ Pacte de stabilité et Semestre européen}

\section{Conclusions du Conseil sur les bilans ap- profondis de 2021}

Le 13 juillet 2021, le Conseil ECOFIN a publié ses conclusions sur les bilans approfondis de 2021 dans le cadre de la procédure concernant les déséquilibres macroéconomiques. En substance, le Conseil considère "qu'il est important de poursuivre la coordination étroite des politiques économiques de l'UE, y compris de détecter, prévenir et corriger les déséquilibres macroéconomiques qui entravent le bon fonctionnement des économies des États membres, de l'Union économique et monétaire ou de l'économie de l'Union européenne dans son ensemble ». II partage l'appréciation de la Commission selon laquelle la crise de la COVID-19 n'a pas fondamentalement changé la nature des déséquilibres des États membres, mais a entraîné un recul dans la réduction des déséquilibres observée précédemment et pourrait accroître les risques pesant sur la stabilité macroéconomique, notamment sur les dettes.

\section{La France veut faire évoluer le pacte de stabilité et de croissance}

Dans la perspective de la présidence française de I'Union européenne à partir de janvier 2022, les sources gouvernementales ont annoncé début novembre vouloir faire évoluer le pacte de stabilité et de croissance.

\section{Politique européenne}

Le Conseil adopte les règlements sur la cohésion pour la période 2021-2027

Le 28 mai 2021, le Conseil a adopté définitivement une série de règlements régissant les Fonds structurels et d'investissement, qui s'élèvent à plus de 330 Mds€ (aux prix de 2018), soit près d'un tiers du budget à long terme de l'UE. Ces fonds financeront classiquement « des projets régionaux et locaux destinés à réduire les disparités économiques et sociales entre États membres et régions", tout en "stimulant une reprise durable après la pandémie grâce à des investissements dans les priorités écologiques et numériques».

NextGenerationEU : adoption d'une stratégie de financement diversifiée

Le 14 avril, la Commission européenne a adopté une stratégie de financement diversifiée pour 
lever, jusqu'en 2026, sur les marchés au meilleur prix les 800 Mds€ d'emprunts prévus par le plan de relance intitulé NextGenerationEU. Les États membres sont invités à présenter leurs plans pour la reprise et la résilience avant le 30 avril, mais ils peuvent le faire à tout moment jusqu'à la mi-2022. En pratique, si quelques-uns ont commencé juste avant (le Portugal en premier le 22 avril, I'Allemagne et la Grèce le 28 avril, la France et la Slovaquie le 29), les autres devraient suivre début mai.

\section{$\pi$ Budget européen}

\section{Mds€ d'obligations vertes NextGene-} rationEU

Après avoir adopté le 7 septembre un cadre pour les obligations vertes, qui garantit aux investisseurs que les fonds mobilisés seront affectés à des projets verts et que la Commission rendra compte de l'impact environnemental de ces projets, la Commission européenne a lancé, le 12 octobre 2021, la première émission d'obligations vertes NextGenerationEU, levant ainsi 12 Mds€ exclusivement destinés à des investissements verts et durables sur tout le territoire de I'Union. Leur montant pourrait atteindre $250 \mathrm{Mds} €$.

\section{L'adoption du budget 2022 de l'Union eu- ropéenne}

Le 24 novembre 2021, le Parlement européen a adopté le budget de I'Union pour 2022 en validant l'accord obtenu le 16 novembre avec le Conseil, fixant le montant total des engagements à 169,5 Mds€ et celui des paiements à 170,6 Mds€

\section{त États-membres}

Allemagne : le débat sur le frein à la dette

En 2020 le gouvernement fédéral avait déjà prévu le creusement du déficit et la mise en suspens du « frein à la dette » (qui restreint les déficits publics à $0,35 \%$ du PIB fédéral), inclus dans la Constitution en 2009. Le débat s'est tendu le 26 janvier quand Helge Braun, ministre fédéral et chef de la chancellerie fédérale a appelé à s'en affranchir pendant de nombreuses années. Son discours empreint de réalisme a suscité l'opposition de plusieurs membres de la CDU, le parti de la chancelière.

La dernière visite de Mme Merkel en Grècere visite de Mme Merkel en Grèce

Le 29 octobre 2021, Mme Merkel est passé faire une ultime visite à Athènes avant de quitter le pouvoir. Elle a reconnu avoir demandé beaucoup à la Grèce lors de la crise des dettes souveraines, ce qui a été interprété par la presse comme une forme «d'auto-critique ». Elle reste, pour cette raison, peu appréciée dans le pays.

\section{Le blocage du plan de relance hongrois}

Le 6 septembre 2021, les ministres de l'économie et des finances ont approuvé les décisions d'exécution des plans pour la reprise et la résilience de la Tchéquie (7 Mds€) et de l'Irlande (989 M€). Tout au long du mois de septembre, plusieurs autres plans nationaux ont été approuvés par la Commission qui a, par ailleurs, versé plusieurs fonds de préfinancement. Mais le plan de relance hongrois, soumis à Bruxelles le 12 mai et qui vise à utiliser les 7,2 Mds€ de subventions européennes auxquels Budapest peut prétendre, aurait dû faire l'objet d'une décision de la Commission le 12 juillet, après deux mois d'examen. La période avait été prolongée jusqu'à fin septembre. Le 17 septembre, le premier ministre hongrois a dénoncé un blocage de la Commission en raison des tensions autour du respect de l'État de droit. De la même façon, le plan de la Pologne n'a pas encore été approuvé et, le 4 septembre, son Premier ministre a déclaré que, si son pays « avait droit » à ces fonds, il se débrouillait très bien sans et «ne sollicitera pas ces fonds en mendiant».

\section{Fiscalité}

Nouveau programme de la Commission en matière de fiscalité des entreprises

Le 18 mai 2021, la Commission européenne a adopté une communication sur la fiscalité des entreprises visant à promouvoir un système fiscal « solide, efficace et équitable pour les entreprises ». Cette communication tient compte des progrès réalisés dans le cadre des discussions menées par le G20 et l'OCDE sur la réforme fiscale mondiale.

\section{Proposition de renouvellement du régime de l'octroi de mer}

Le 3 mars 2021, la Commission a proposé de renouveler le régime fiscal spécial des régions ultrapériphériques françaises de l'UE pour la période 2022-2027.

\section{Mise à jour de la liste des paradis fiscaux}

Le 5 octore 2021, dans des conclusions, le Conseil a décidé de retirer Anguilla, la Dominique et les Seychelles de la liste de I'UE des pays et territoires non coopératifs à des fins fiscales. II reste neuf noms sur la liste de l'UE des pays et territoires non coopératifs: les Samoa américaines, les Fidji, Guam, les Palaos, le Panama, le Samoa, 
Trinité-et-Tobago, les Îles Vierges américaines et le Vanuatu.

\section{INTERNATIONAL}

\section{$\lambda$ États}

\section{États-Unis : le plan de relance est adopté \\ Le 10 mars 2021, le président Biden a obtenu du Congrès l'adoption de son plan de relance éco- nomique de 1900 Mds\$ Mds\$. Le Sénat l'avait adopté le samedi 6 mars (par 50 voix contre 49) et la Chambre des représentants I'a suivi par 220 voix contre 211. Ce montant « colossal » équi- vaut au PIB de I'Italie. Le plan comprend notam- ment des chèques d'aides directes allant jusqu'à 1400 dollars par individu et personne à charge, pour un montant global de quelque 400 Mds\$.}

\section{Londres détrônée par Amsterdam comme place financière}

Dans un article paru le 10 février, le Financial Times constate que Londres a été détrônée. Amsterdam devient la première place financière européenne en volume d'échanges boursiers en récupérant les transactions «perdues par le Royaume-Uni depuis le Brexit ». Près de 9,2 Mds€ d'actions ont été négociés chaque jour à la Bourse d'Amsterdam en janvier, «soit quatre fois plus qu'en décembre ", contre 8,6 Mds€ pour la City, selon des données de CBOE Europe, I'une des principales plateformes de négociation d'actions européennes.

\section{Relations multilatérales}

UE-États-Unis : suspension du conflit aérien

Le 5 mars 2021, L'Union européenne et les ÉtatsUnis ont convenus de suspendre, pour une période de quatre mois, toutes les mesures tarifaires de rétorsion imposées sur les exportations européennes et américaines dans le cadre des différends liés à Airbus et Boeing.

\section{$\pi$ Fiscalité}

L'accord sur l'imposition minimale des multinationales et ses conséquences pour les GAFAMM

Le vendredi 8 octobre 2021, l'accord définitif sur une taxation des multinationales au taux de
$15 \%$ été signé par 136 pays, dont l'Irlande qui a fini, avec l'Estonie, par s'y rallier la veille. L'Inde a accepté ce texte in extremis. Il été validé au sommet du G20 à Rome, le 30 octobre (cf. Rubrique Fiscalité). Dans la foulée de cet accord, I'administration américaine a demandé à I'UE d'abandonner son projet de taxe sur les GAFAM, pourtant prévue pour financer le remboursement du plan de relance. Le gel de cette taxe, annoncé dès le 12 juillet, ne simplifie par le dossier des ressources propres européennes. D'autant que la Secrétaire d'État au Trésor, estime qu'Amazon ne sera pas soumise totalement à la future taxe, faute de dégager dans toutes ses activités segmentées le surprofit de $10 \%$ qui en sera la condition d'application.

\section{$\pi$ Monnaies}

\section{Cryptomonnaies : le SEC américain veut} réguler le " Far West »

Le 3 août 2021, 3 août, le nouveau patron de la Securities and Exchange Commission (SEC, le "gendarme» boursier américain), Gary Gensler a déclaré vouloir réguler les cryptomonnaies en visant en priorité les plates-formes d'échange, et $c e$, afin de mieux protéger les investisseurs.

\section{La Chine juge illégales toutes les transac- tions en cryptomonnaies}

Le 24 septembre 2021, La Banque centrale de Pékin a déclaré illégales toutes les transactions financières impliquant des monnaies virtuelles. Elle souhaite désormais ainsi lutter contre le "blanchiment d'argent, [les] collectes de fonds illégales, la fraude, et d'autres activités illégales et criminelles ». Cette déclaration a fortement perturbé le cours, très fluctuant de toute façon, des cryptomonnaies, notamment celui du bitcoin.

\section{Le retour de l'inflation : les réactions des Banques centrales}

Le mercredi 15 décembre 2021, la Réserve fédérale a décidé de réagir plus nettement face à l'inflation. Elle a annoncé prévoir trois hausses des taux directeurs pour 2022. Ils passeront ainsi en moyenne à $0,9 \%$ fin 2022 , puis à $1,6 \%$ fin 2023 avec deux hausses prévues dans l'année, puis à $2,1 \%$ en 2024 avec deux hausses également et enfin $2,5 \%$ à long terme.

Robin Degron 